\title{
Nanocrystalline silicon: lattice dynamics and enhanced thermoelectric properties
}

Cite this: Phys. Chem. Chem. Phys. 2014, 16, 25701

Received 4th September 2013, Accepted 8th May 2014

DOI: $10.1039 / c 3 c p 53749 h$

www.rsc.org/pccp

\author{
Tania Claudio, ${ }^{\text {ab }}$ Niklas Stein, ${ }^{c}$ Daniel G. Stroppa, ${ }^{d}$ Benedikt Klobes, ${ }^{a}$ \\ Michael Marek Koza, ${ }^{\mathrm{e}}$ Petra Kudejova, ${ }^{\mathrm{C}}$ Nils Petermann, ${ }^{\mathrm{C}}$ Hartmut Wiggers, ${ }^{\mathrm{C}}$ \\ Gabi Schierning ${ }^{c}$ and Raphaël P. Hermann*ab
}

\begin{abstract}
Silicon has several advantages when compared to other thermoelectric materials, but until recently it was not used for thermoelectric applications due to its high thermal conductivity, $156 \mathrm{~W} \mathrm{~K}^{-1} \mathrm{~m}^{-1}$ at room temperature. Nanostructuration as means to decrease thermal transport through enhanced phonon scattering has been a subject of many studies. In this work we have evaluated the effects of nanostructuration on the lattice dynamics of bulk nanocrystalline doped silicon. The samples were prepared by gas phase synthesis, followed by current and pressure assisted sintering. The heat capacity, density of phonons states, and elastic constants were measured, which all reveal a significant, $\approx 25 \%$, reduction in the speed of sound. The samples present a significantly decreased lattice thermal conductivity, $\approx 25 \mathrm{~W} \mathrm{~K}^{-1} \mathrm{~m}^{-1}$, which, combined with a very high carrier mobility, results in a dimensionless figure of merit with a competitive value that peaks at $Z T \approx 0.57$ at $973{ }^{\circ} \mathrm{C}$. Due to its easily scalable and extremely low-cost production process, nanocrystalline Si prepared by gas phase synthesis followed by sintering could become the material of choice for high temperature thermoelectric generators.
\end{abstract}

\section{Introduction}

Silicon is the second-most abundant element in the Earth's crust after oxygen. Due to its abundance and widespread usage in the semiconductor industry, the material itself and also precursors for gas phase synthesis are available in industrial quantities, at exceptional purity and quality for a comparatively low price. Si is non-toxic and unproblematic from a safety, scarcity and environmental perspective, unlike other, lead or tellurium bearing, thermoelectric materials. Further, the entire technology around silicon - synthesis, machining, etching, doping - is well-established and it is one of the most studied and simplest model materials in solid state physics, for which reference data is widely available. ${ }^{1} \mathrm{Si}$ is therefore a material of choice to study the effects of nanostructuration on the lattice

${ }^{a}$ Jülich Centre for Neutron Science JCNS and Peter Grünberg Institut PGI, JARA-FIT, Forschungszentrum Jülich GmbH, D-52425 Jülich, Germany.

E-mail: r.hermann@fz-juelich.de; Fax: +49 (0) 246161 2610; Tel: +49 (o) 2461614786

${ }^{b}$ Faculté des Sciences, Université de Liège, B-4000 Liège, Belgium

${ }^{c}$ Faculty of Engineering and Center for Nano-integration Duisburg-Essen (CENIDE), University of Duisburg-Essen, D-47057, Duisburg, Germany

${ }^{d}$ Peter Grünberg Institut PGI-5, Forschungszentrum Jülich GmbH, D-52425 Jülich, Germany

${ }^{e}$ Institut Laue Langevin, 6 rue Jules Horowitz B.P. 156, F-38042 Grenoble, France

${ }^{f}$ Technische Universitt München, Forschungsneutronenquelle Heinz Maier-Leibnitz (FRM II), Lichtenbergstr. 1, D-85747 Garching, Germany dynamics and the thermal transport, which must be mastered in order to achieve good thermoelectric properties.

Single-crystalline and undoped silicon has a very high thermal conductivity, $156 \mathrm{~W} \mathrm{~K}^{-1} \mathrm{~m}^{-1}$ at room temperature, ${ }^{2}$ related to its low density and high Young's modulus. In heavily doped silicon, the thermal conductivity is significantly reduced, though still very high, close to $80 \mathrm{~W} \mathrm{~K} \mathrm{~K}^{-1} \mathrm{~m}^{-1}$, at room temperature. ${ }^{3}$ As a direct result of its lattice structure with stiff tetrahedral covalent bonds connecting the atoms, it is a hard but brittle material.

Nanostructuration as means to improve the thermoelectric properties of a material has been intensively studied, ${ }^{4}$ since such processing decreases the lattice thermal conductivity by creating additional scattering centers for phonons at grain boundaries. By influencing mostly the lattice contribution to the thermal conductivity without also decreasing the electronic contribution, which is directly related to the electrical conductivity, an improvement in the dimensionless thermoelectric figure of merit $(Z T)$ - and ultimately the efficiency of a thermoelectric generator - can be achieved, since $Z T=S^{2} \sigma T / \kappa$, where $S$ is the Seebeck coefficient, $\sigma$ the electrical conductivity, $\kappa$ the thermal conductivity and $T$ the temperature. Such approach is viable because phonons have usually a mean free path significantly larger than electrons, and the transport of the former can be disrupted without dramatically hindering the latter.

The effects of nanostructuration on the lattice dynamics were investigated by means of theoretical calculations ${ }^{5-12}$ and 
experimentally observed by methods such as inelastic neutron scattering, ${ }^{13-18}$ Raman spectroscopy, ${ }^{19,20}$ nuclear inelastic scattering (NIS) ${ }^{17,21}$ and measurements of the specific heat. ${ }^{22,23}$ Overall, these calculations and experiments reveal that an enhancement in the density of phonon states (DPS) at low energies and a broadening of the bands on the DPS is expected for nanocrystalline materials. These modifications in the vibrational modes are attributed to the vibrations of atoms located at the grain boundaries where the atomic structure is more open than within the crystalline grains, and result in a modified force field and a softening of the force constants.

Here we present a detailed experimental study on the effects of nanostructuration on the lattice dynamics and thermoelectric properties of nanocrystalline silicon which, except for the intentional doping, is virtually free of other impurities.

\section{Experimental}

\subsection{Sample preparation}

Silicon nanoparticles were synthesized by a plasma-assisted gas phase process, using a microwave reactor as described previously. ${ }^{24} 1 \%$ of $n$-type doping was achieved by adding phosphine $\left(\mathrm{PH}_{3}\right)$ to the precursor gas. Two batches of powder were obtained by controlling the synthesis parameters: one with a nominal initial grain size of $14 \mathrm{~nm}$ and another with a nominal initial grain size of $52 \mathrm{~nm}$ confirmed by BET. $\dagger$ The nanopowder is functionalized at the surface by hydrogen during the synthesis, so that no oxygen impurities can attach at the surface of freshly synthesized powder. The nanopowder was bottled under inert conditions and kept within an inert atmosphere to prevent contamination with oxygen during further processing. Sintering was carried out immediately after synthesis with an absolute minimum time for filling the crucible and starting the sintering process. This effort is necessary, as a contamination of the nanopowder with oxygen can significantly influence the physical properties of the samples as reported previously. ${ }^{25}$

The nanopowder was compacted into $2 \mathrm{~cm}$ diameter dense pellets with a spark plasma sintering furnace from FCT Systeme $\mathrm{GmbH}$ in a $1 \mathrm{mbar}$ Ar atmosphere. For the first batch $(14 \mathrm{~nm})$, sintering was carried out during $3 \mathrm{~min}$ (sample A). The second batch $(52 \mathrm{~nm})$ was divided into two parts. One part was sintered for $3 \mathrm{~min}$ (sample $\mathrm{B}$ ) and the other for $30 \mathrm{~min}$ (sample $\mathrm{C}$ ). Heating and cooling rates for all samples were fixed to $100 \mathrm{~K} \mathrm{~min}^{-1}$. The sintering temperature was $1150{ }^{\circ} \mathrm{C}$ and a $35 \mathrm{MPa}$ pressure was applied during sintering.

\subsection{Microstructural characterization and composition}

Transmission Electron Microscopy (TEM) was used for structural characterization of the sintered pellets in a FEI-Tecnai microscope with a field-emission electron gun operating at $200 \mathrm{kV}$. TEM samples were prepared from the sintered pellets by a precision Focused Ion Beam (FIB) system using gallium ions. The grain size estimation was carried out measuring 75 grains on each sample in different regions. As the probe volume

$\dagger$ BET (Brunauer, Emmett, Teller): surface area analysis. is small, $\sim 0.1$ cubic micron, the microstructure was also investigated by scattering methods.

In order to verify the average crystallite size of the nanocrystalline pellets, X-Ray diffraction (XRD) was performed using synchrotron radiation at the high energy station 6-ID-D of the Advanced Photon Source (APS) at Argonne National Laboratory. The sample was $1 \mathrm{~mm}$ thick and the experiment was performed in transmission geometry in order to probe the bulk of the pellets. The X-ray wavelength was $0.124659 \AA$ and a General Electric amorphous silicon detector was positioned at a distance of $1849 \mathrm{~mm}$ from the sample, a distance determined by a NIST640c Si standard. The data were reduced to diffraction patterns with the program FIT2 $\mathrm{D}^{26}$ and no preferential orientation was observed. Rietveld refinements were carried out using the program FULLPROF, ${ }^{27}$ taking into consideration the Debye-Scherrer broadening of the diffraction peaks.

The average crystallite size was also investigated by Small Angle Neutron Scattering (SANS) measurements carried out using the instrument KWS1 operated by the Jülich Centre for Neutron Science (JCNS) at the FRM II (Garching, Germany). ${ }^{28}$ In the present work, only the essential results concerning characteristic crystallite sizes will be presented, while further analysis based on Gaussian random fields ${ }^{29}$ will be published elsewhere.

The elemental analysis was performed at the Prompt Gamma Ray Activation Analysis (PGAA) instrument positioned at the neutron guide NL4b of the Forschungs-Neutronenquelle Heinz Maier-Leibnitz (FRM II - Garching, Germany). ${ }^{30}$ The cold neutron flux used for the measurement was $6.1 \times 10^{10} \mathrm{n} \mathrm{cm}^{-2} \mathrm{~s}^{-1}$. A high-purity germanium (HPGe) detector was used to detect the gamma rays produced in the sample by neutron capture reactions. The emitted gamma rays have a characteristic energy which depends on the element or isotope which absorbs the neutrons. The peak-area is proportional to the concentration of the element in the sample. This method is therefore a noninvasive way to chemically characterize the sample, which gives precise information about the impurities and its amount in percentages, and it is a method of choice which can give nondestructively information about elements such as hydrogen and boron.

\subsection{Lattice dynamics}

Resonant Ultrasound Spectroscopy (RUS) is a nondestructive technique which gives access to the elastic tensor by recording the frequencies of the natural modes of mechanical vibrations. Parallelepiped samples are positioned between two piezoelectric transducers, one driving and the other recording the response, ${ }^{31}$ in order to obtain the elastic constants $C_{11}$ and $C_{44}$ that fully characterize the elasticity in an elastically isotropic samples. The first 30 resonance frequencies were measured on samples with $\sim 2.0 \times 1.5 \times 1.5 \mathrm{~mm}^{3}$ dimensions. The RMS errors between the calculated and measured frequencies were $0.29 \%$ and $0.21 \%$ for the samples with smaller and larger nanocrystallites, respectively.

The Density of Phonon States (DPS) of all samples was obtained by inelastic neutron scattering measurements performed on the Time of Flight (TOF) spectrometer IN6 at the 
cold source of the Institute Laue-Langevin (Grenoble, France). The incident wavelength was $5.12 \AA$ with an elastic energy resolution of $0.13 \mathrm{meV}$, as determined by the elastic neutron scattering on a vanadium sample.

The specific heat, $C_{\mathrm{p}}$, was obtained in a commercial Physical Property Measurement System (PPMS) from Quantum Design for all sintered pellets between 4 and $400 \mathrm{~K}$. The specific heat of a polycrystalline Si sample was also measured for comparison.

\subsection{Thermoelectric transport measurements}

Thermoelectric transport characterization was carried out between room temperature and $1000{ }^{\circ} \mathrm{C}$. The Seebeck coefficient $S$ and the specific electrical conductivity $\sigma$ were measured on a bar-shaped sample by a direct measurement technique using a commercial system (ZEM3 by Ulvac Technologies, Inc.). In contrast to ref. 32, we did not carry out a rapid thermal annealing to $1000{ }^{\circ} \mathrm{C}$. However, all measurements were carried out upon slow heating at a rate between 1.5 and $2.5 \mathrm{~K} \mathrm{~min}^{-1}$ in order to warrant comparison. The thermal diffusivity $\lambda_{\theta}$ was measured by a laser flash method on a sample of $10 \times 10 \times 1 \mathrm{~mm}^{3}$ size using a commercial system (LFA 457 MicroFlash by Netzsch Thermal Analysis $\mathrm{GmbH}$ ). The thermal conductivity was then calculated with $\kappa=\lambda_{\Theta} \cdot C_{\mathrm{p}} \cdot \rho$, using a value for the density of the pellets $(\rho)$ obtained by the Archimedes method at room temperature. The bulk values of heat capacity were used for this calculation, since typically in this temperature range the heat capacity of bulk and nanocrystalline $\mathrm{Si}$ does not differ, as confirmed from the $C_{\mathrm{p}}$ results. In addition, charge carrier densities were determined at room temperature from Hall effect measurements carried out in the classical Hall bar geometry with magnetic fields ranging between -1 and $1 \mathrm{~T}$ and using alternating currents.

\section{Results and discussion}

\subsection{Microstructural and chemical characterization}

The average nanocrystallite diameters, $D$, as well as the strain in the sintered pellets was estimated by refinement of the X-ray diffraction pattern and the results were compared with images obtained from TEM, see Fig. 1(a) and (b), and with the results of the SANS data shown in Fig. 2 as a Kratky plot of the scattering intensity, $\mathrm{d} \Sigma / \mathrm{d} \Omega \cdot Q^{2}$ vs. $Q$, where $Q$ is the scattering vector. The Kratky plot exhibits a maximum at $q_{\text {max }}$, which can be used to estimate the average crystallite diameter $D$ using the pseudoGuinier radius $R_{\mathrm{pg}}=D / 2=\sqrt{3} / q_{\max } \cdot{ }^{33}$ The values obtained by these three methods are summarized in Table 1.

Sample A had an average crystallite size of $40(2) \mathrm{nm}$ from XRD and $42 \mathrm{~nm}$ from SANS whereas TEM reveals grains ranging between 48 and $264 \mathrm{~nm}$ with an average of $114 \mathrm{~nm}$. Sample C had a slightly smaller crystallite size of 33(1) and $34 \mathrm{~nm}$ from $\mathrm{XRD}$ and SANS, respectively, and a grain size distribution obtained by TEM between 47 and $246 \mathrm{~nm}$ with an average of $112 \mathrm{~nm}$, and also showed amorphous precipitates with average size of $29 \mathrm{~nm}$. The differences seen between TEM, SANS and XRD analysis have their origin in sensitivity of the different methods. TEM is sensitive to the outer contour of grains, not
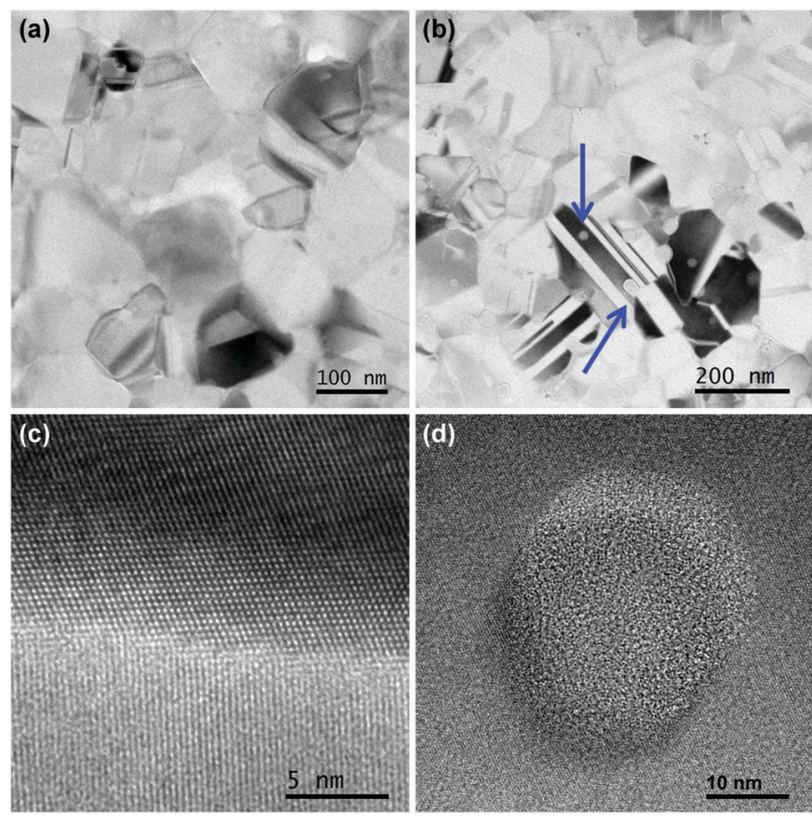

Fig. 1 TEM bright-field images of sample A (a) and sample $C$ (b) and high resolution TEM of grain boundary between two nanocrystals of sample $A$ (c) and of an amorphous precipitates on sample C (d). Arrows indicate amorphous precipitates.

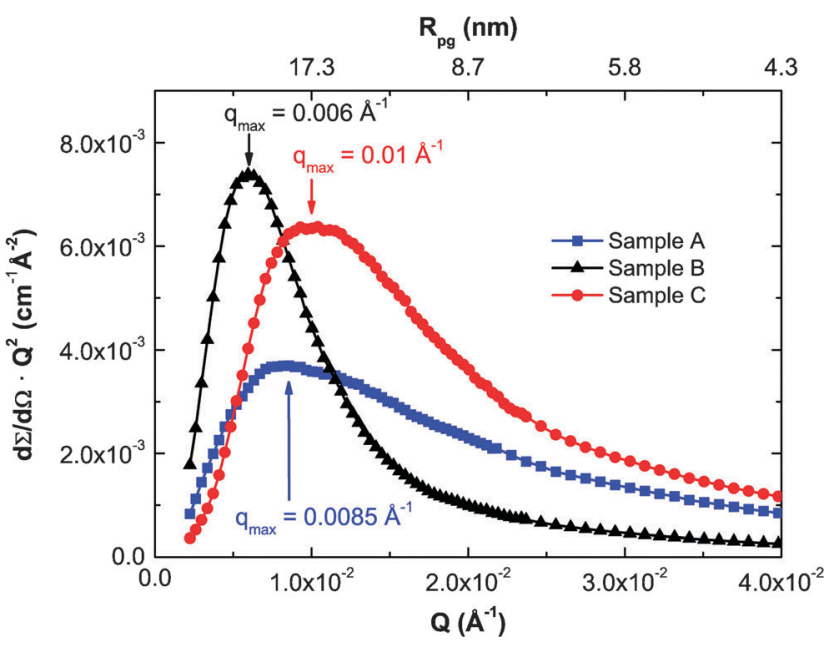

Fig. 2 Kratky plot of the SANS curves. Each curve exhibits a broad maximum, which yields the pseudo-Guinier radius $R_{\mathrm{pg}}$

Table 1 Summary of nanocrystallite sizes $(D)$ and strain $(\varepsilon)$ obtained by TEM analysis, XRD refinement and SANS

\begin{tabular}{|c|c|c|c|c|c|c|}
\hline & \multicolumn{3}{|l|}{$\underline{\text { TEM }}$} & \multicolumn{2}{|l|}{ XRD } & \multirow{2}{*}{$\frac{\text { SANS }}{D(\mathrm{~nm})}$} \\
\hline & $D_{\min }(\mathrm{nm})$ & $D_{\max }(\mathrm{nm})$ & $D_{\mathrm{av}}(\mathrm{nm})$ & $D(\mathrm{~nm})$ & $\varepsilon(\%)$ & \\
\hline Sample A & 48 & 264 & 114 & $40(2)$ & 0.00138 & 42 \\
\hline Sample B & - & - & - & $42(2)$ & 0.00133 & 58 \\
\hline Sample C & 47 & 246 & 112 & $33(1)$ & 0.00150 & 34 \\
\hline
\end{tabular}

taking into account possible twinning or intragranular effects. $\mathrm{XRD}$ is sensitive to the crystallite size, i.e. to limited correlation length, which is in general smaller than the grain size due to the 
existence of planar defects and intra grain boundaries. Furthermore, SANS curves strongly depend on the size distribution of the scattering entities and may also be affected by inter-particle effects in the present case, though it still represents a measure of characteristic length scales. ${ }^{33}$ Besides, XRD and SANS are methods averaging over a larger volume, whereas TEM only gives an impression of a very small sample volume, not necessarily fully representative for the complete pellet. Correlating the different methods and known behavior of nanocrystalline silicon, the presence of significant amounts of an amorphous cover of silicon, in excess of one or two atomic layers, can be ruled out, in particular because of the large charge mobility, see below, and because a change in contrast would be noticeable at the grain boundaries next to regions in diffracting condition and would appear black in bright field TEM images.

The samples which were processed with the same sintering parameters, but with initially different nanoparticles sizes (14 and $52 \mathrm{~nm}$ for samples A and C, respectively), present only a small different average nanocrystallite sizes after sintering. Sample A presented strong grain growth during sintering. Sample C presented a large amount of defects such as twins and a certain amount, $\sim 6 \%$, of amorphous precipitates, which energy-dispersive X-ray spectroscopy indicated to be essentially $\mathrm{Si}$, both visible in Fig. 1(b), resulting in an increased strain as obtained from XRD refinement. A grain boundary between two nanocrystals in sample A with different relative orientation without any amorphous layer is shown in Fig. 1(c) and a high resolution image of an amorphous precipitate on sample $\mathrm{C}$ in Fig. 1(d). Those precipitates are rather spherical and are found on all images acquired on this sample, indicated by blue arrows in Fig. 1(b).

Overall, although the three different measurements yielded different values of nanocrystallite sizes, the same trend in sizes is observed, ranging from the smallest being sample $\mathrm{C}$ and the largest being sample B.

Samples B and C were investigated exemplarily by PGAA. The spectra reveal that the samples were $99.0(1) \%$ Si with $1.0(7) \%$ of $\mathrm{P}$ dopant as expected. A small amount of $\mathrm{H}(0.20(1) \%)$ was detected on the sample with smaller initial nanocrystallite size (sample C). Furthermore, in both samples, a very small ppm contribution of boron was detected. This impurity could originate from a contamination of the precursor silane, which is prepared by fractional distillation, as it is not easy to completely separate silane from diborane.

\subsection{Lattice dynamics}

Samples A and B had the same properties in all measurements within their experimental errors: density of phonon states

Table 2 Summary of the elastic constants and sound velocities in nanocrystalline Si compared with bulk Si obtained by different techniques

\begin{tabular}{llllll}
\hline & & & \multicolumn{2}{l}{$v_{\mathrm{s}}\left(\mathrm{km} \mathrm{s}^{-1}\right)$} \\
\cline { 5 - 6 } & $C_{11}(\mathrm{GPa})$ & $C_{44}(\mathrm{GPa})$ & RUS & DPS & $C_{\mathrm{p}}, \Theta_{\mathrm{D}}^{\mathrm{LT}}$ \\
\hline Bulk & $160.1^{34}$ & $80.0^{34}$ & 5.94 & $6.73(5)$ & $5.70(5)$ \\
Sample A & $173(3)$ & $59.0(8)$ & $5.0(1)$ & $5.0(3)$ & $4.44(9)$ \\
Sample C & $172(3)$ & $58.2(7)$ & $4.9(1)$ & $4.3(3)$ & $4.2(1)$
\end{tabular}

(DPS), specific heat $\left(C_{\mathrm{p}}\right)$ and resonant ultrasound spectroscopy (RUS). Therefore, only the results for sample A are being discussed here.

The values obtained for the elastic constants $C_{11}$ and $C_{44}$ with RUS are summarized in Table 2 . The speed of sound was then calculated: $3 / v_{\mathrm{s}}{ }^{3}=1 / v_{\text {long }}{ }^{3}+2 / v_{\text {trans }}{ }^{3}$, where $v_{\text {long }}=\sqrt{C_{11} / \rho}$ and $v_{\text {trans }}=\sqrt{C_{44} / \rho}$ for the polycrystals are the longitudinal and transversal speed of sound, respectively. The values of speed of sound for single-crystalline Si were calculated previously (ref. 25) using the Hershey-Kröner-Eshelby average.

The elastic constant $C_{11}=172(3)$ and 173(3) GPa for the samples with smaller and larger nanocrystallites sizes, respectively, corresponding to the bulk modulus $(B)$ is $\approx 8 \%$ larger than in single crystalline Si. The shear modulus, $G=C_{44}=58.2(7)$ and $59.0(8) \mathrm{GPa}$, is $\sim 25 \%$ smaller than in single crystalline $\mathrm{Si}$. Such a decrease in the resistance to shear deformation $(G)$ combined with an increase of the resistance to dilation $(B)$ shows that the mechanical properties of a material with as many grain boundaries and defects are significantly modified when compared with the single-crystalline material, leading to a softening of the material. This is also confirmed by the decrease of the speed of sound calculated from the elastic constants. Such a decrease relates to a decrease in thermal conductivity.

The DPS of two samples of nanocrystalline Si obtained from inelastic neutron scattering are shown in Fig. 3 and compared with polycrystalline $\mathrm{Si}^{25}$ Note that the area under the DPS curve of all samples was normalized to 1 between 0 and $70 \mathrm{meV}$.

In the incoherent scattering approximation, and because the sample essentially contains only one chemical element, the speed of sound can be obtained from the low energy limit of $g(E) / E^{2}$ with $1 / v_{s}^{3}=2 \pi^{2} N_{V} / \hbar^{3} g(E) / E^{2}$ where $N_{V}$ is the number of atoms per unit volume. ${ }^{17}$ In the case of the nanocrystalline $\mathrm{Si}$ samples an increase on the Debye level, $\lim _{E \rightarrow 0} g(E) / E^{2}$, in the reduced DPS is observed. Note that the incoherent scattering approximation is not perfectly suitable for silicon, which is a strong coherent scatterer. Therefore, the speed of sound is

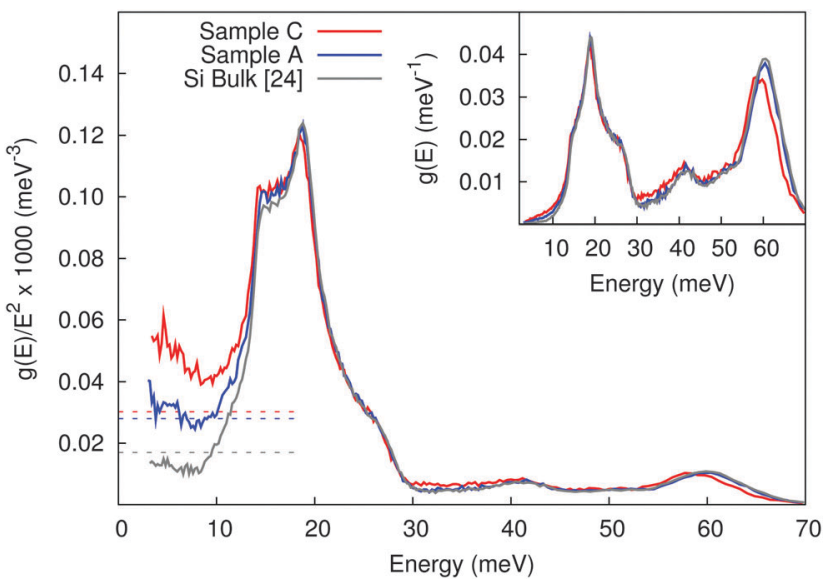

Fig. 3 Reduced Density of Phonon States (DPS) for two samples of nanocrystalline Si compared to previously reported results on polycrystalline Si, dotted lines indicate the asymptotic limits from RUS. Inset: DPS. 


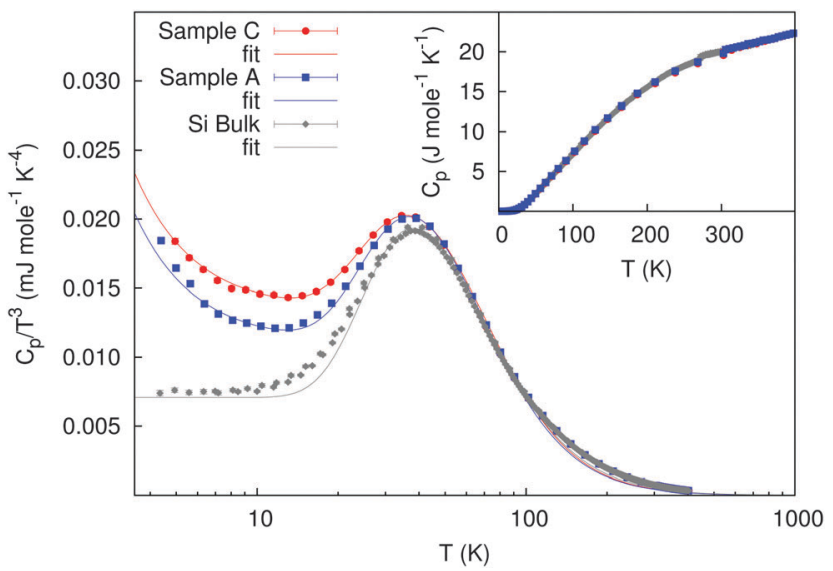

Fig. 4 Specific heat divided by $T^{3}$ for both nanocrystalline Si compared with polycrystalline Si. Inset: specific heat (error bars are smaller than the symbol size).

obtained by a second approach using RUS measurements, and the data is compared. An extrapolation using the asymptotic limit from RUS, considering the speed of sound summarized in Table 2, shown as dotted lines in Fig. 3, reveals a fair agreement of both methods.

On one hand, the sample containing a small amount of grain boundaries (sample A) presented the same results of speed of sound calculated from the reduced DPS and obtained with RUS, with only a small part of the reduced DPS data being above the asymptotic limit from RUS. On the other hand, the reduced DPS of the sample with larger amount of grain boundaries, defects, and amorphous contribution (sample C) is significantly above the asymptotic limit from RUS and has a pronounced increase for $E \rightarrow 0$. We therefore conclude that the excess states in the reduced DPS, which is probed only above $3 \mathrm{meV}$, are above the asymptotic limit and can be considered as contribution due to the larger amount of grain boundaries, defects and amorphous inclusions.

The specific heat, represented as $C_{\mathrm{p}} / T^{3} v s$. $T$, of the nanocrystalline silicon samples measured between 4 and $400 \mathrm{~K}$ compared to bulk $\mathrm{Si}$ is shown in Fig. 4. The nanocrystalline samples have the same $C_{\mathrm{p}}$ at high temperatures as bulk Si. Below $30 \mathrm{~K}$ an increased lattice contribution, related to the increased Debye level and decreased speed of sound, is observed. Further, an electronic contribution, linear in temperature, and thus $\propto 1 / T^{2}$ in $C_{\mathrm{p}} / T^{3}$, is observed at the lowest temperatures, because with such high carrier concentrations, in the \% range, there is no freeze-out of the charge carriers as would be expected in semiconductors.
At the lowest temperatures, a simple model of the heat capacity can be designed by considering an electronic contribution $(\gamma T)$ and a lattice contribution that can be described using the Debye model and an Einstein term to describe the excess $C_{\mathrm{p}}$ at approximately $45 \mathrm{~K}:^{25}$

$$
C_{\mathrm{p}}(T)=\gamma T+d C_{\mathrm{D}}(T)+e C_{\mathrm{E}}(T)
$$

where $d$ and $e$ are prefactors for the Debye and Einstein contributions, $C_{\mathrm{D}}(T)$ and $C_{\mathrm{E}}(T)$, respectively. More details about the Debye and Einstein models can be found in ref. 35-37. A fit to the data up to $90 \mathrm{~K}$ yields parameters as summarized in Table 3. The electronic contribution $\left(\gamma \approx 0.1 \mathrm{~mJ} \mathrm{~mol}^{-1} \mathrm{~K}^{-2}\right)$ is close to that obtained on the specific heat of metals (0.6-2.5 mJ mol $\left.{ }^{-1} \mathrm{~K}^{-2}\right),{ }^{36}$ and shows a significant presence of electronic contribution in the samples. The values obtained for the Einstein and Debye temperatures were also converted to energy $(1 \mathrm{~K}=0.0862 \mathrm{meV})$ and the Einstein energies are in very good agreement with the feature observed at this energy ( $\approx 16 \mathrm{meV}$ ) in the reduced DPS (Fig. 3 ). The Debye temperature obtained from a fit in this temperature range $\left(\Theta_{\mathrm{D}}^{\mathrm{HT}}\right)$ differs from the temperature obtained considering only the low temperature Debye plateau $\left(\Theta_{\mathrm{D}}^{\mathrm{LT}}\right)$, as it probes higher energies phonon modes. For the calculations of the speed of sound from the specific heat, $v_{\mathrm{s}}=k_{\mathrm{B}} \Theta_{\mathrm{D}}^{\mathrm{LT}} / \hbar\left(6 \pi^{2} N_{V}\right)^{1 / 3}$, only the Debye plateau was considered, and yields values as summarized in Table 2 .

\subsection{Thermoelectric and transport measurements}

The general trend of the electrical conductivity $(\sigma)$ of all samples between room temperature and $750{ }^{\circ} \mathrm{C}$ demonstrates a decrease with temperature as expected for degenerately doped semiconductors (Fig. 5(a)). This occurs due to the increase of the lattice vibrations in this range of temperature, i.e. electron scattering by phonons dominates the observed temperature trend, as in metals. Above this temperature an additional contribution is observed leading to a kink in $\sigma$ which increases with temperature above temperatures of $750{ }^{\circ} \mathrm{C}$. Such a kink in a n-type semiconductor might a priori be attributed to bipolar contributions, ${ }^{38}$ when additional p-type charge carriers are thermally activated over the band gap of the semiconductor, but this explanation does not hold for silicon, which has a too large band gap. An alternative explanation for a similar kink previously observed on nanocrystalline Si by Bux et al., ${ }^{32}$ is the kinetics of dopant precipitation related to the retrograde solid solubility of phosphorus in silicon. ${ }^{39}$ As the temperature rises, additional phosphorus atoms are being incorporated at substitutional lattice sites and become electrically activated. This leads to a rapid increase in carrier concentration and

Table 3 Values of the electronic contribution $(\gamma)$, Einstein and Debye temperatures as well as heat capacity prefactors $(e$ and $d)$ obtained with a fit of the eqn (1) for low temperatures (below $90 \mathrm{~K}$ )

\begin{tabular}{|c|c|c|c|c|c|c|c|}
\hline & \multirow[b]{2}{*}{$\gamma\left(\mathrm{mJ} \mathrm{mol}{ }^{-1} \mathrm{~K}^{-2}\right)$} & \multirow[b]{2}{*}{$e\left(\mathrm{~J} \mathrm{~mol}^{-1} \mathrm{~K}^{-1}\right)$} & \multicolumn{2}{|l|}{$\underline{\Theta_{\mathrm{E}}}$} & \multirow[b]{2}{*}{$d\left(\mathrm{~J} \mathrm{~mol}^{-1} \mathrm{~K}^{-1}\right)$} & \multicolumn{2}{|l|}{$\underline{\Theta}_{\mathrm{D}}^{\mathrm{HT}}$} \\
\hline & & & $(\mathrm{K})$ & $(\mathrm{meV})$ & & $(\mathrm{K})$ & $(\mathrm{meV})$ \\
\hline Bulk & - & $3.8(3)$ & $188(2)$ & $16.2(2)$ & $11.4(3)$ & $501(17)$ & $43(2)$ \\
\hline Sample C & $0.12(2)$ & $2.02(3)$ & 184(1) & 15.9(1) & $11.2(9)$ & $402(11)$ & $34.6(9)$ \\
\hline
\end{tabular}



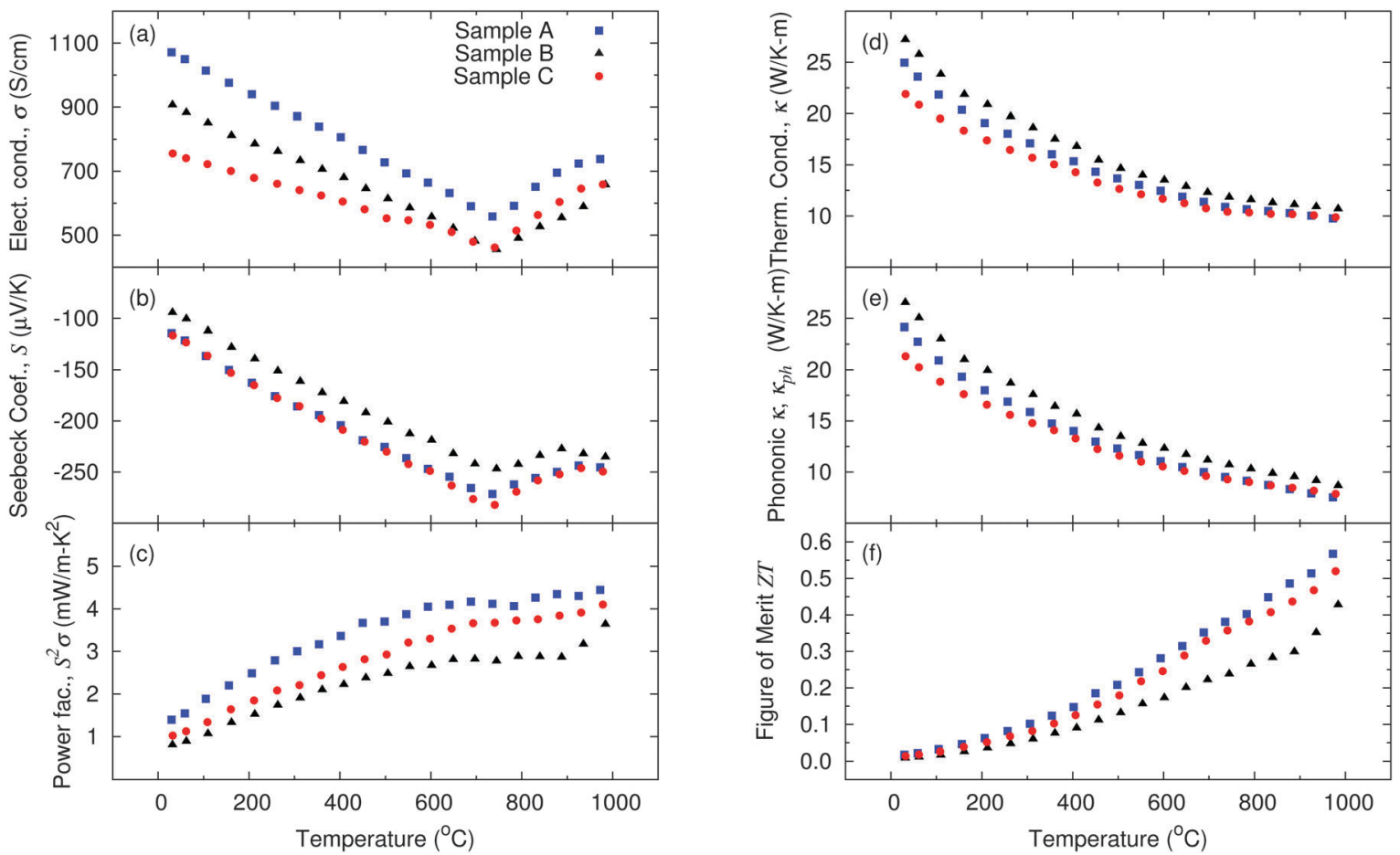

Fig. 5 Thermoelectric characterization of the 3 nanocrystalline Si samples: (a) electrical conductivity; (b) Seebeck coefficient; (c) calculated power factor; (d) thermal conductivity; (e) calculated lattice contribution to the thermal conductivity and (f) calculated dimensionless figure of merit.

consequently an increase of the electrical conductivity as well as a decrease of the Seebeck coefficient. This is possible because at low temperature less phosphorus atoms are electrically activated than chemically incorporated. The tendency towards hysteresis ${ }^{39}$ in the heating and cooling cycles confirms dopant kinetic as an explanation of this kink rather than the bipolar effect.

The Si sample with smaller nanocrystallite sizes presents a lower electrical conductivity between room temperature and $750{ }^{\circ} \mathrm{C}$ when compared with the two other samples. The sample which was sintered for a longer period of time has lower $\sigma$ than the sample with large nanocrystallites sintered for a short time.
Both samples which were sintered for a short period of time presented similar values of the Seebeck coefficient (Fig. 5(b)), i.e., likely a very similar charge carrier concentration. The sample with smaller nanocrystallites presented a lower $\sigma$ than the sample with larger crystallites, due to a large amount of grain boundaries, defects and amorphous contribution leading to a more pronounced scattering of electrons. This results in a better power factor for the sample with large nanocrystallites, but sintered for a short period of time (Fig. 5(c)).

Although the sample which was sintered for a longer period of time (sample B) had a slightly higher density when compared with the other samples (only 1.1\% higher, see in Table 4), its

Table 4 Summary of room temperature properties for all nanocrystalline Si samples, compared with previously published results on spark plasma sintered bulk nanocrystalline $\mathrm{Si}$ and single-crystal Si

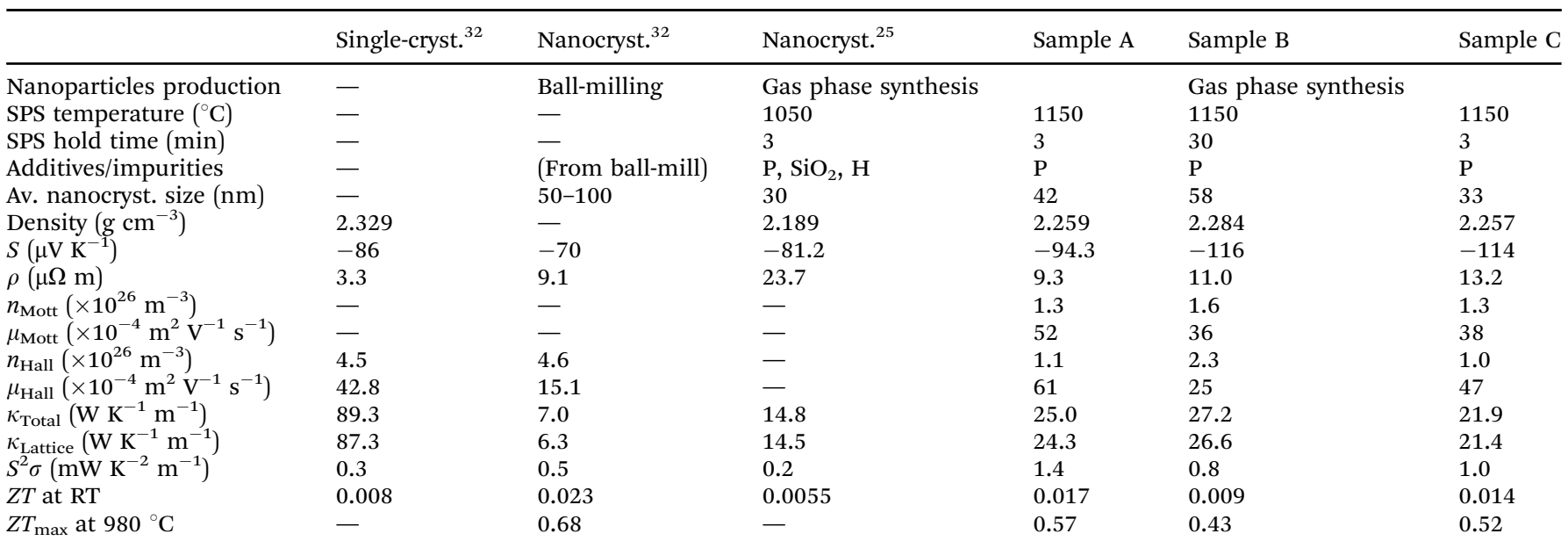


electronic properties were not better. During the long sintering period (30 minutes) at high temperatures $\left(1150{ }^{\circ} \mathrm{C}\right)$, diffusion processes occur. Those do not only lead to a healing of defects, which rather improve the crystalline quality of the sample as seen by higher values of thermal conductivity (Fig. 5(d)) and by the higher density, but also to diffusion of the impurities (dopants). The latter have a tendency to aggregate on grain boundaries. This agglomeration of dopants can be seen in the weak increase in $\sigma$ for $T>750{ }^{\circ} \mathrm{C}$. Overall, the power factor of this sample is lower than for the two samples sintered within only 3 minutes (samples A and C), see Fig. 5(c).

The charge carrier concentration $(n)$ can be estimated from the slope of the Seebeck coefficient (for $T<750{ }^{\circ} \mathrm{C}$ ): ${ }^{38}$

$$
S=\frac{8 \pi^{2} k_{\mathrm{B}}^{2}}{3 e h^{2}} m^{*} T\left(\frac{\pi}{3 n}\right)^{2 / 3}
$$

where $k_{\mathrm{B}}$ is the Boltzmann constant, $h$ is the Planck constant, $e$ is the elementary charge and $m^{*}=1.13 m_{e, 0}{ }^{40}$ is the reduced band effective mass of electrons in silicon. Band transport in one parabolic band as well as an energy independent scattering time were assumed to derive this relatively simple transport model from the Mott equation, ${ }^{41}$ strictly valid only in metals but useful also for the analysis of degenerated doped semiconductors. A comparison between Hall data and an evaluation of the Seebeck coefficient for nanocrystalline silicon showed a decent agreement for electron mobility and charge carrier concentration, ${ }^{42}$ so that this simple approximation is used here to compare nano silicon transport data. From the combined measurement of the Seebeck coefficient and the electrical conductivity, we estimated the mobility of the charge carriers, $\mu$, with $\sigma=n e \mu$. In addition, as the Mott equation is not strictly valid for doped semiconductors, we have determined the charge carrier density in sample $\mathrm{A}$ and $\mathrm{B}$ from the Hall coefficient, $R_{\mathrm{H}}, n=1 / R_{\mathrm{H}} e$ assuming a Hall factor value of 1 . The obtained charge carrier densities from the latter method are in reasonable agreement with those obtained from the Mott formula. The values obtained are summarized in Table 4 and compared with literature data. The difference between the lattice thermal conductivity and total thermal conductivity, i.e. the electronic contribution, $\kappa_{\mathrm{e}}$, has been estimated using $\kappa_{\mathrm{e}}=L T / \rho$, where $L=2.2 \times 10^{-8} \mathrm{~W} \Omega \mathrm{K}^{-2}$ is the estimated Lorentz factor for highly doped $\mathrm{Si}^{32}$ The mobility obtained for the samples under investigation is larger than in heavily doped bulk single crystalline silicon, ${ }^{32}$ which might seem surprising. However, as the mobility is dependent on the charge carrier concentration and decreases with increasing concentration at very large doping level, ${ }^{43}$ this observation is reasonable.

Comparing the samples produced from different nanoparticle batches (14 nm vs. $52 \mathrm{~nm}$ ), but sintered for the same short period of time ( 3 minutes), no significant decrease of the thermal conductivity was observed, since sample A showed a more pronounced grain coarsening during sintering. The sample with initially smaller nanocrystallite size has a thermal conductivity $\kappa$ which is $12 \%$ lower at room temperature than the sample with initially larger nanocrystallite size, but at high temperatures the thermal conductivity data of both samples converges.
The lattice contribution to the thermal conductivity accounts for up to $97 \%$ of the total thermal conductivity at room temperature and decreases to around $77 \%$ at the highest measured temperature. In single crystalline silicon, $90 \%$ of the heat is transported by phonons with mean free path larger than $100 \mathrm{~nm} .{ }^{44}$ Therefore, an average grain size around $100 \mathrm{~nm}$ or slightly smaller is already effective in lowering the lattice thermal conductivity as can be seen from the investigated samples.

The thermal conductivity of the pure nanocrystalline samples studied in this paper is still large when compared with previously reported results on nanocrystalline $\mathrm{Si}^{25,32,42}$ but in those samples, several impurities were present either from exposing the nanopowder to air before sintering ${ }^{25,42}$ or due to additives used for nanopowder preparation with ball-milling. ${ }^{32}$ These impurities may significantly affect the mobility of charge carriers, $\mu$, leading to a smaller power factor for the samples in earlier reports than for the samples discussed herein, see Table 4. Next to impurities, a second possible cause for both the higher mobility and thermal conductivity for the samples investigated here, is the fabrication method. Where the bottom up gas phase synthesis followed by sintering is expected to lead to well crystallized particles, the top down approach using ballmilling $^{32}$ is more prone to lead to a strained lattice, and thus, smaller mobility but also smaller thermal conductivity.

Overall, despite the large thermal conductivity, the dimensionless figure of merit of the silicon nanocomposites investigated herein still reaches competitive values due to a very high carrier mobility resulting in comparatively high power factors. With a $Z T$ of 0.57 at $973{ }^{\circ} \mathrm{C}$, the sample produced with $52 \mathrm{~nm}$ nanoparticles and sintered for 3 minutes can compete with other results published so far, ${ }^{32}$ with the advantage of a more easily scalable production process.

\section{Conclusions}

The three different nanocrystalline Si samples which were analyzed in this work showed interesting lattice dynamics and thermoelectric properties depending on their synthesis parameters. Both samples which were prepared with the same nanoparticle size, but sintered for different periods of time, presented same results on the study of their lattice dynamics: a decrease in the speed of sound when compared to singlecrystalline $\mathrm{Si}$. The sample which was sintered for a longer period (30 $\mathrm{min}$ ) showed also a decrease in the thermoelectric properties with a lower power factor and an elevated thermal conductivity due to improved crystallinity.

The sample with small initial nanoparticle size showed a significant grain growth during sintering as analyzed by XRD refinement and TEM, and also a larger amount of defects and a certain amount of amorphous precipitates, leading to a further decrease on the speed of sound and to an excess of vibration modes at low energies. It also presented slightly worse electronic properties, which compensates the lower thermal conductivity and leads to a similar dimensionless figure of merit as the 
sample prepared with larger nanoparticles and same sintering parameters.

A systematic trend in the decrease of the speed of sound obtained from the density of phonon states, resonant ultrasound spectroscopy, and specific heat, combined with the large amount of grain boundaries in nanostructured Si materials, resulted in a thermal conductivity four times lower than for a single-crystal.

When compared with previously reported results on nanocrystalline $\mathrm{Si}$, the samples still present a somewhat large thermal conductivity, which is compensated by a very high power factor and results in competitive values of the dimensionless figure of merit with a peak $Z T$ of 0.57 at $973{ }^{\circ} \mathrm{C}$. When compared to other materials which are used in this temperature range such as nanocrystalline SiGe, Si has a $Z T$ which is approximately half of the results reported so far (1.3 at $900{ }^{\circ} \mathrm{C}^{45}$ ), with the advantage of being approximately 14 times cheaper than SiGe.

Since the thermal conductivity of the nanocrystalline Si samples presented in this work is still large when compared to other thermoelectric materials, further optimization of the parameters leading to the reduction of the thermal conductivity could lead to an even better $Z T$ value.

Furthermore, studies have shown that the energy cost of Si nanopowder production through gas phase synthesis is drastically reduced with increasing production amount. ${ }^{46}$ Therefore, when compared with other methods such as ball-milling, gas phase synthesis has the great advantage of being a continuous and a more easily scalable method.

\section{Acknowledgements}

The Institute Laue-Langevin (ILL - Grenoble, France) and the Advanced Photon Source (APS) at Argonne National Laboratory are acknowledged for neutron and synchrotron radiation beam time respectively. The Helmholtz Gemeinschaft Deutscher Forschungszentren is acknowledged for funding VH NG-407 "Lattice dynamics in emerging functional materials". We would like to thank Dr Z. Di (JCNS, Forschungszentrum Jülich, outstation at FRM II) for support during beam time. Dr D. Bessas is kindly acknowledged for collecting the PGAA data, D. Meertens for TEM sample preparation, Dr D. Weber for scientific input and Dr K. Friese for input on XRD refinement. The Deutsche Forschungsgemeinschaft, DFG, is acknowledged for funding through the program SPP1386 "Nanostukturierte Thermoelektrika".

\section{References}

1 Landolt-Börnstein - Group III Condensed Matter, ed. O. Madelung, U. Rössler and M. Schulz, SpringerMaterials The Landolt-Börnstein Database, 2011, ch. Silicon (Si), vol. 41.

2 C. J. Glassbrenner and G. A. Slack, Phys. Rev., 1964, 134, A1058-A1069.

3 H. R. Shanks, P. D. Maycock, P. H. Sidles and G. C. Danielson, Phys. Rev., 1963, 130, 1743-1748.
4 M. S. Dresselhaus, G. Chen, M. Y. Tang, R. G. Yang, H. Lee, D. Z. Wang, Z. F. Ren, J. P. Fleurial and P. Gogna, Adv. Mater., 2007, 19, 1043-1053.

5 D. Wolf, J. Wang, S. Phillpot and H. Gleiter, Phys. Lett. A, 1995, 205, 274-280.

6 S. Phillpot, J. Wang, D. Wolf and H. Gleiter, Materials Science \& Engineering A (Structural Materials: Properties, Microstructure and Processing), Div. of Mater. Sci., Argonne Nat. Lab., IL, USA, 1995, pp. 76-82.

7 M.-S. Jeng, R. Yang, D. Song and G. Chen, J. Heat Transfer, 2008, 130, 042410.

8 R. Singh and S. Prakash, Indian J. Phys., A, 2003, 77, 243-246.

9 R. Singh, S. Prakash, R. Meyer and P. Entel, Pramana J. Phys., 2003, 60, 547-556.

10 A. I. Potapov, I. S. Pavlov and S. A. Lisina, J. Sound Vib., 2009, 322, 564-580.

11 J. Wang, D. Wolf, S. R. Phillpot and H. Gleiter, Nanostruct. Mater., Div. of Mater. Sci., Argonne Nat. Lab., IL, USA, 1995, pp. 747-750.

12 S. Stankov, M. Miglierini, A. I. Chumakov, I. Sergueev, Y. Z. Yue, B. Sepiol, P. Svec, L. Hu and R. Rüffer, Phys. Rev. B: Condens. Matter Mater. Phys., 2010, 82, 144301.

13 B. Fultz, C. C. Ahn, E. E. Alp, W. Sturhahn and T. S. Toellner, Phys. Rev. Lett., 1997, 79, 937-940.

14 H. Frase, B. Fultz and J. L. Robertson, Phys. Rev. B: Condens. Matter Mater. Phys., 1998, 57, 898-905.

15 S. Mentese, J.-B. Suck and A. J. Dianoux, Mater. Sci. Forum, Inst. fur Phys., Tech. Univ. Chemnitz, Germany, 2000, pp. 671-676.

16 S. Mentese, J.-B. Suck and S. Janssen, Physica B, Inst. of Phys., Mater. Res. \& Liquids, Technische Univ. Chemnitz, Germany, 2002, pp. 438-440.

17 A. I. Chumakov, A. Bosak and R. Rüffer, Phys. Rev. B: Condens. Matter Mater. Phys., 2009, 80, 094303.

18 L. Saviot, C. H. Netting, D. B. Murray, S. Rols, A. Mermet, A.-L. Papa, C. Pighini, D. Aymes and N. Millot, Phys. Rev. B: Condens. Matter Mater. Phys., 2008, 78, 245426.

19 D. Bersani, P. P. Lottici and X. Z. Ding, Appl. Phys. Lett., 1998, 72, 73-75.

20 M. Maczka, M. Ptak, M. Kurnatowska, L. Kȩpiński, P. Tomaszewski and J. Hanuza, J. Solid State Chem., 2011, 184, 2446-2457.

21 S. Stankov, Y. Z. Yue, M. Miglierini, B. Sepiol, I. Sergueev, A. I. Chumakov, L. Hu, P. Svec and R. Rüffer, Phys. Rev. Lett., 2008, 100, 235503.

22 B. Haiyang, L. Jianlin, J. Duo and S. Jirong, Chin. Phys. Lett., 1995, 12, 549-552.

23 L. Wang, Z. Tan, S. Meng, A. Druzhinina, R. A. Varushchenko and G. Li, J. Non-Cryst. Solids, 2001, 296, 139-142.

24 N. Petermann, N. Stein, G. Schierning, R. Theissmann, B. Stoib, M. S. Brandt, C. Hecht, C. Schulz and H. Wiggers, J. Phys. D: Appl. Phys., 2011, 44, 174034.

25 T. Claudio, G. Schierning, R. Theissmann, H. Wiggers, H. Schober, M. M. Koza and R. P. Hermann, J. Mater. Sci., 2013, 48, 2836-2845. 
26 A. P. Hammersley, FIT2D: An Introduction and Overview, ESRF ESRF Internal Report ESRF97HA02T, 1997.

27 J. Rodriguez-Carvajal, FULLPROF V (2009), Laboratoire leon brillouin (cea-cnrs), france technical report, 2009.

28 A. Radulescu and A. Ioffe, Nucl. Instrum. Methods Phys. Res., Sect. A, 2008, 586, 55-58.

29 J. A. Quintanilla and W. M. Jones, Phys. Rev. E: Stat., Nonlinear, Soft Matter Phys., 2007, 75, 046709.

30 L. Canella, P. Kudějová, R. Schulze, A. Türler and J. Jolie, Nucl. Instrum. Methods Phys. Res., Sect. A, 2011, 636, 108-113.

31 A. Migliori and J. L. Sarrao, Resonant Ultrasound Spectroscopy: Applications to Physics, Materials Measurements, and Nondestructive Evaluation, Wiley-Interscience, 1st edn, 1997.

32 S. K. Bux, R. G. Blair, P. K. Gogna, H. Lee, G. Chen, M. S. Dresselhaus, R. B. Kaner and J.-P. Fleurial, Adv. Funct. Mater., 2009, 19, 2445-2452.

33 A. Deschamps and F. De Geuser, J. Appl. Crystallogr., 2011, 44, 343-352.

34 Landolt-Börnstein - Group III Condensed Matter, ed. O. Madelung, U. Rössler and M. Schulz, SpringerMaterials - The LandoltBörnstein Database, 2011, ch. Silicon (Si) elastic moduli of Si-I, vol. 41A1a.

35 R. P. Hermann, F. Grandjean and G. J. Long, Am. J. Phys., 2005, 73, 110-118.
36 C. Kittel, Introduction to Solid State Physics, Wiley, New York, 7th edn, 1995.

37 N. W. Ashcroft and N. D. Mermin, Solid State Physics, Thomson Learning, Inc., 1976.

38 A. J. Minnich, M. S. Dresselhaus, Z. F. Ren and G. Chen, Energy Environ. Sci., 2009, 2, 466-479.

39 R. Olesinski, N. Kanani and G. Abbaschian, Bull. Alloy Phase Diagrams, 1985, 6, 130-133.

40 M. A. Green, J. Appl. Phys., 1990, 67, 2944-2954.

41 G. J. Snyder and E. S. Toberer, Nat. Mater., 2008, 7, 105-114.

42 G. Schierning, R. Theissmann, N. Stein, N. Petermann, A. Becker, M. Engenhorst, V. Kessler, M. Geller, A. Beckel, H. Wiggers and R. Schmechel, J. Appl. Phys., 2011, 110, 113515.

43 Physics of Semiconductor devices, ed. S. M. Sze and K. K. Ng, John Wiley and Sons, 3rd edn, 2007, p. 29.

44 C. Dames and G. Chen, in Thermoelectrics Handbook: Macro to Nano, ed. D. M. Rowe, CRC/Taylor \& Francis, Boca Raton, FL, 2006, vol. 1, ch. 42, pp. 42-10-42-16.

45 X. W. Wang, H. Lee, Y. C. Lan, G. H. Zhu, G. Joshi, D. Z. Wang, J. Yang, A. J. Muto, M. Y. Tang, J. Klatsky, S. Song, M. S. Dresselhaus, G. Chen and Z. F. Ren, Appl. Phys. Lett., 2008, 93, 193121-193123.

46 T.-P. Hüser, S. M. Schnurre, H. Wiggers and C. Schulz, KONA Powder Part. J., 2011, 29, 191-207. 